\title{
Active control of coupled wave propagation in fluid-filled elastic cylindrical shells
}

\author{
B. J. Brévart and C. R. Fuller \\ Vibration and Acoustics Laboratories, Mechanical Engineering Department, Virginia Polytechnic Institute \\ and State University, Blacksburg, Virginia 24061-0238
}

(Received 24 June 1992; revised 9 February 1993; accepted 24 May 1993)

\begin{abstract}
A control approach to reduce the total power propagating along fluid-filled elastic cylinders is analytically investigated. The motion of the cylinder is described by the Kennard shell equations fully coupled to the interior acoustic field. The vibration disturbance source is a pre-determined free propagating wave of either $n=0$ or $n=1$ circumferential order and the control forces considered are appropriate harmonic line forces radially applied to the structure. The radial displacement of the shell wall at discrete locations downstream of control forces is minimized using feedforward quadratic optimal theory. The difference of total power flow through the system before and after control is then used to evaluate the impact of the fluid on the performance of the control approach. For the breathing circumferential mode $(n=0)$, owing to the coupling between the two media, the fluid decreases the control performance when the disturbance is a structural-type incident wave. When the disturbance is a fluid-type incident wave, with a pressure near field concentrated at the shell wall, significant reductions of the transmitted power flow can be achieved. For the beam mode $(n=1)$, even though the control is applied to the structure, the fluid increases the control performances below the first acoustic cut-off frequency and decreases it above this frequency.
\end{abstract}

PACS numbers: $43.40 . \mathrm{Vn}, 43.40 . \mathrm{Ey}$

\section{LIST OF SYMBOLS}

$a$

$c_{f}$

$\boldsymbol{c}_{\boldsymbol{F}_{\text {sj }}}$

$F_{c}$

FL

$F_{0}$

$h$

$J_{n}()$

$k_{n}$

$k_{n s}$

$\left(k_{n s} a\right)_{\text {inc }}$

$k_{s}^{r}$

$n$

$p$

$p_{0}$

$P_{f}$

$P_{n s}$

$P_{s}$

$r, x, \theta$

$R_{a}$

$s$

$S_{n}$ thin shell mean radius

fluid acoustic free wave speed

shell extension phase speed

fluid power factor

control force

fluid loading term

amplitude of the input force

shell wall thickness

Bessel function of order $n$

axial wave number

axial wave number of the branch $s$

axial wave number of the incident wave

radial wave number

circumferential mode number

pressure

input force distribution

power flow in the fluid field

pressure amplitude

shell power flow

cylindrical coordinates

ratio of axial to radial amplitude

branch number

number of propagating branches for a cir-

cumferential mode $n$

$S_{s j n}^{f}$
$\mathrm{TL}$
$T_{p}$
$u, v, w$
$U_{n s}, V_{n s}, W_{n s}$
$\bar{U}_{n}, \bar{V}_{n}, \bar{W}_{n}$
$w_{n s}^{\text {inc }}$
$W_{n s}^{\text {inc }}$
$x_{0}$
$x_{i}$
$\beta$
$\delta$
$\delta$
$\Delta X_{f}$
$\Delta X_{p}$
$\epsilon$
$\eta$
$v$
$\rho_{f}$
$\rho_{s}$
$\omega$
$\Omega$

shell power factor

power transmission loss

transmission power coefficient

shell displacements

shell displacement amplitudes

shell spectral displacement amplitudes

disturbance wave displacement

disturbance wave amplitude

position of the input force

position where the disturbance complex

amplitude is $1+0 i$

thickness factor

Dirac delta function

partial derivative

distance between control forces

distance between error points

$=2$ if $n=0,=1$ if $n \neq 0$

damping coefficient

Poisson's ratio

density of fluid

density of shell

frequency

nondimensional shell frequency, $\mathbf{\Omega}$

$=\omega a / c_{L}$

\section{INTRODUCTION}

Vibration of fluid-filled cylinders is an important problem in many industrial and defense applications. Piping systems can be excited by a large number of sources related either to the structural or the internal fluid path, through

disturbances such as compressors, pumps, or valves. More important is the fact that, as vibrational energy propagates along a piping system, it can excite other equipment attached to the structure, often at distances far from the source. Therefore, it is of prime importance to be able to 
control the total energy flow going through these systems. Due to the coupling between the structure and the fluid, this can be a difficult task to achieve by passive methods. The present paper investigates an active control approach to reduce the vibration and hopefully the total power flow through an infinite fluid-filled elastic cylindrical shell. In addition, it would be preferable to apply the active control directly to the shell wall even though the aim is to control both the shell and the fluid power. Such a configuration would be less obtrusive on the flow field and easier to install. A thorough understanding of the dynamic behavior of fluid-filled shells is critical to an investigation on active control applied to such systems. Fuller and Fahy ${ }^{1}$ have analytically studied the pipe behavior in terms of propagation of free waves and gav" physical interpretations of the eigenvalues of the infinite system. They ${ }^{1}$ also determined whether the vibrational energy was located in the pipe wall or the contained fluid. More recently, Brévart and Fuller ${ }^{2}$ evaluated the effect of an internal uniform flow on this distribution of vibrational energy. Fuller also considered the problem of struccural excitation by a radial line force ${ }^{3}$ and internal monopole excitation ${ }^{4}$ of the coupled system. Leyrat and Cushieri ${ }^{5}$ presented a solution for the mobility of a cylindrical shell system subjected to a radial line force including the effect of a uniform internal how. Finally, Fuller and Brevart ${ }^{6} \mathrm{r} c e n t l y$ investigated the determination of energy propagation paths into a fluid-filled eiastic cylindrical shell excitec by a radial mpulsive line force applied to the shell wall.

In the present paper, the total power flow reduction in the coupled pipe-fid system, dive to the active control if the radial displacement of the ripe wall, will be present $-d$ considering harmonic structural.type and fluid-type incident free waves as disturbances $i n$ both $n=0$ and $n=1$ circumferential modes. One or is $s$ harmonic line forces radially applied on the pipe wall will be used as control forces. The error information 10 be minimiced will be the radial displacement of the shel ar one or two locations, depending on the number of coniryl forces. Resuits will be presented in comparison to the stive control approach performed on an in vacuo shell.

\section{CONTROL METHODOLOGY}

The cylindrical coordinate system employed in the theory is shown in Fig. 1. The diagrams in Fig. 2 describe the arrangements of the two control approaches investigated. In both cases, we consider as a disturbance on the infinite cylinder, an incident propagating fice wave solution $s$ of circumferential order $n$. This wave is written in terms of shell radial displacement as

$$
w_{n s}^{\mathrm{inc}}(x, t)=W_{n s}^{\mathrm{inc}} \exp \left(i\left\{\left(k_{n s} a\right)_{\mathrm{inc}}\left[\left(x+x_{i}\right) / a\right]\right\}-i \omega t\right),
$$

where the nondimensional axial wave number $\left(k_{n s} a\right)_{\text {inc }}$ is given by the dispersion curves of the system for this particular mode $n$. The disturbance has amplitude $W_{n s}^{\text {inc }}$ at $x=-x_{i}$. Due to added damping (see later), the amplitude will slightly decay as the wave propagates through the control discontinuity.
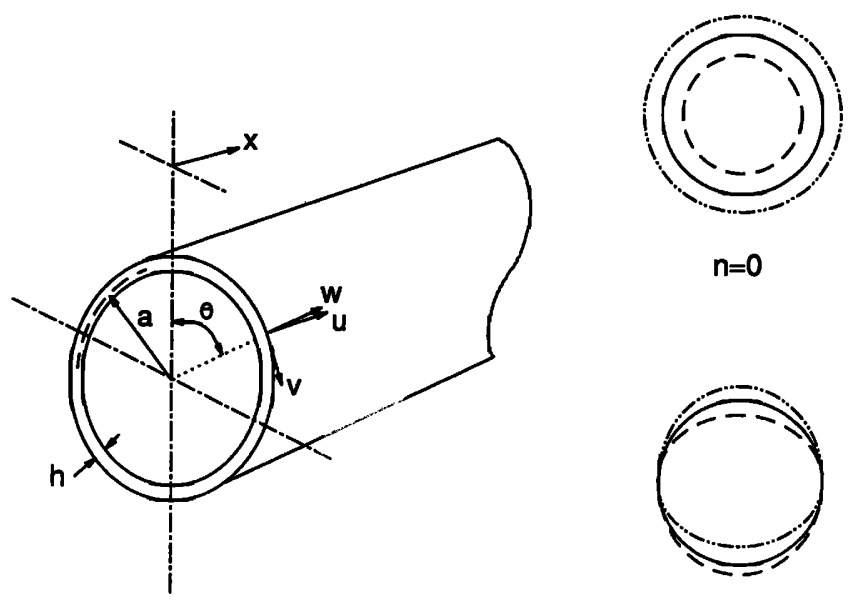

$n=1$

FIG. 1. Coordine ie system and modal shapes.

\section{A. Input disturbance, free wave propagation}

The equation of motion and eigenvalues of the infinite system for free wave propagation have been derived and their physical interpretation discussed in Ref. 1. In this reference, Fuller used the Donnell-Mushtari shell equations to describe the shell motion. These equations are a simplified version of the Kennard's differential equations and were recently found to give unsatisfactory results at extremely low frequencies. Kennard's equations are thus prefered in this investigation. Figure 3(a) and (b) shows

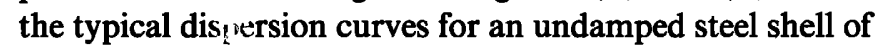
thickness $h / a=0.05$ in vacuo vibrating in the $n=0$ and $n=1$ circumferential modes derived using the Kennard equations. Figure 4(a) and (b) shows the corresponding dispersion curves when the shell is filled with water.

Considering first the breathing mode $(n=0)$ and an in vacuo shell, there is only one wave [see Fig. 3(a)] which is purely real and thus propagating at all frequencies and it is denoted as the branch $s=1$ (a very small imaginary part appears if some structural damping is introduced in the
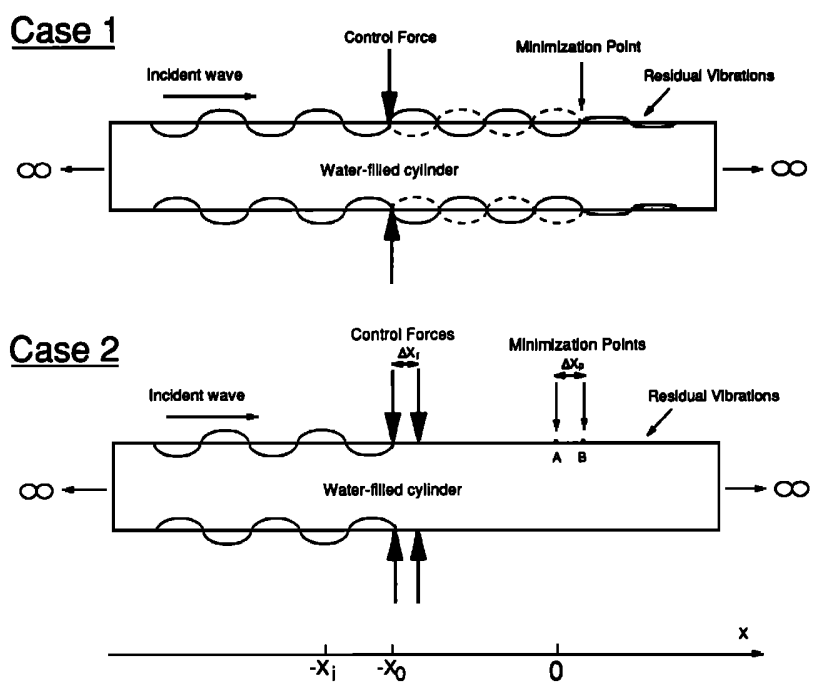

FIG. 2. Control system configurations. 

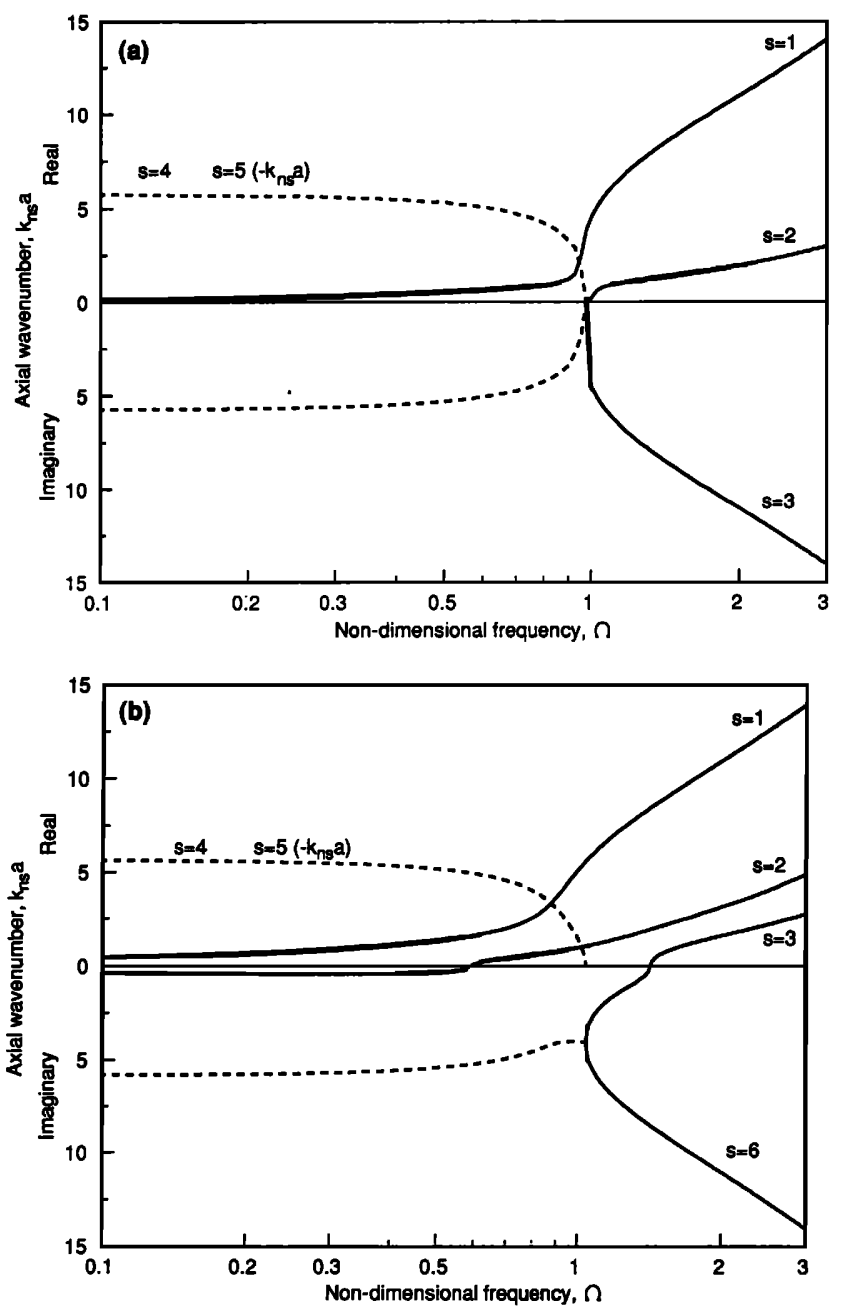

FIG. 3. Dispersion curves of an in vacuo steel shell, $h / a=0.05$; - - purely real and purely imaginary $k_{n s} a$;---, real and imaginary parts of complex $k_{n s} a$; (a) $n=0$, (b) $n=1$.

system). This wave is purely extensional in nature at low frequencies and changes into a flexural wave around the ring frequency $\Omega=1$ (Ref. 7). If the shell is now filled with water [Fig. 4(a)], two waves denoted as $s=1$ and 2, propagate at all frequencies and can be used as incident disturbances, since both of them are purely real roots. The branch $s=1$ is an "acoustically slow" wave, close in nature to a fluid wave in a rigid-walled tube at low frequencies. This branch approaches the in vacuo flexural solution at very high frequencies. Its main characteristic is that the radial wave number, at all frequencies, is purely imaginary. This implies that, in the fluid field, the nature of the wave is a pressure near field concentrated close to the shell wall. To be more precise, the fluid loading for this particular wave appears as a mass loading added on the inner side of the structure. The branch $s=2$ is a structural-type wave at low frequencies, close to the in vacuo extensional solution. Because of the heavy fluid introduced in the system and the induced coupling phenomenon, this branch turns into a pressure release duct solution above the ring frequency.

Considering the beam mode $(n=1)$, both dispersion curves for the in vacuo and the water-filled shell [Figs. 3(b)
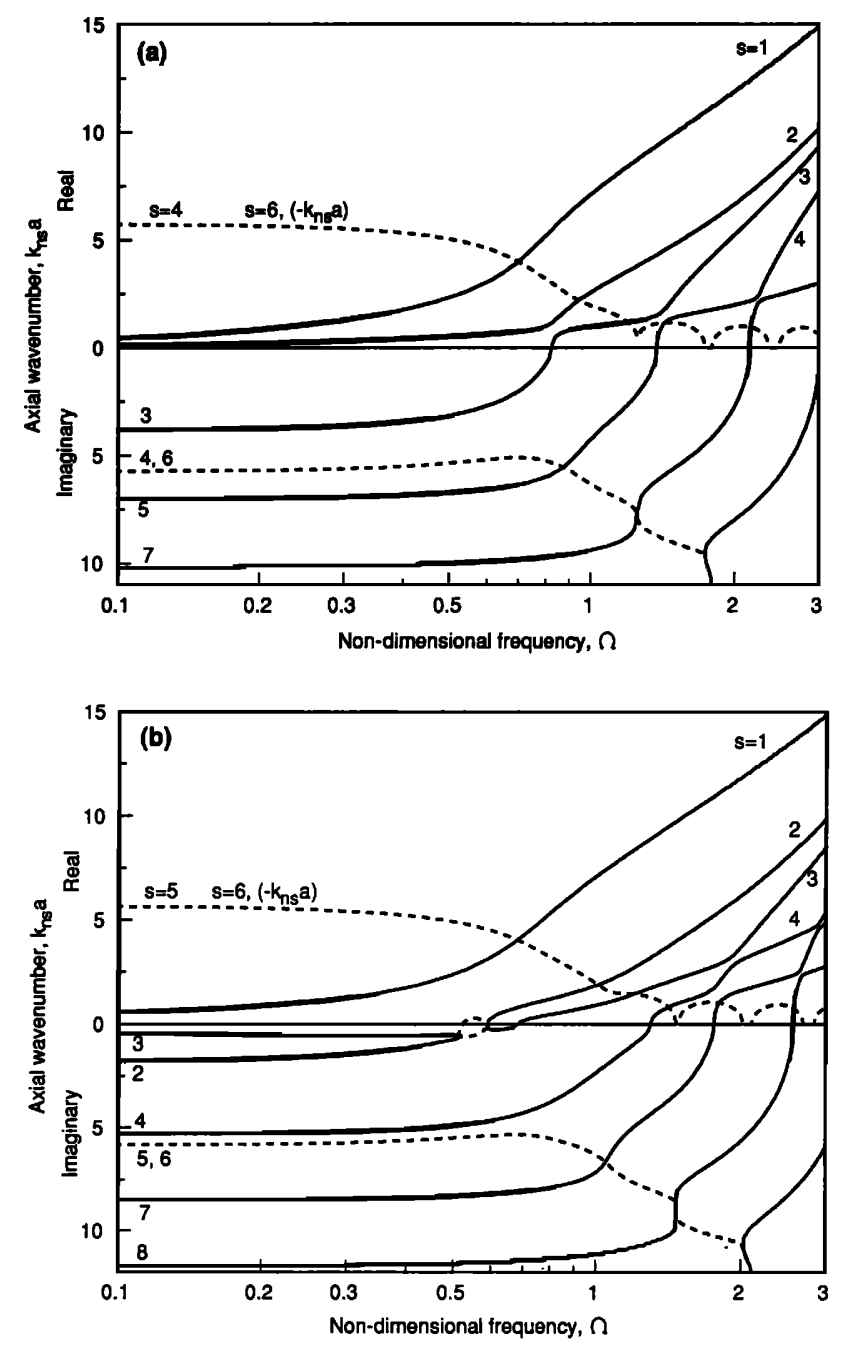

FIG. 4. Dispersion curves of a water-filled steel shell, $h / a=0.05$;- - purely real and purely imaginary $k_{n s} a ;----$, real and imaginary parts of complex $k_{n s} a$; (a) $n=0$, (b) $n=1$.

and 4(b)], display only one purely real wave at low frequencies; the response is similar to the flexural vibrations of a rod at low frequencies and the main effect of the fluid is to increase the mass of the system. Thus, only one kind of disturbance has to be considered for the circumferential mode $n=1$.

\section{B. Forced vibrations}

The control forces considered are radial line forces of the same circumferential distribution as the disturbance applied to the pipe wall (see Fig. 2):

$$
p_{0}(x, t)=F_{0} \cos (n \theta) \delta\left(x+x_{0}\right) e^{-i \omega t}
$$

where $F_{0}$ is the force per unit length of circumference. The input mobility of an infinite fluid-filled cylindrical shell has been previously derived by Fuller. ${ }^{3}$ The shell displacements for the circumferential mode $n$ are first expressed as inverse Fourier transforms,

$$
u=\frac{1}{\sqrt{2 \pi}} \int_{-\infty}^{+\infty} \bar{U}_{n} \cos (n \theta) e^{i\left(k_{n} x-\omega t-\pi / 2\right)} d k_{n}
$$




$$
\begin{aligned}
& v=\frac{1}{\sqrt{2 \pi}} \int_{-\infty}^{+\infty} \bar{V}_{n} \sin (n \theta) e^{i\left(k_{n} x-\omega t\right)} d k_{n}, \\
& w=\frac{1}{\sqrt{2 \pi}} \int_{-\infty}^{+\infty} \bar{W}_{n} \cos (n \theta) e^{i\left(k_{n} x-\omega t\right)} d k_{n} .
\end{aligned}
$$

Substituting these relations into the Kennard shell equations, $^{8}$

$$
\begin{aligned}
& \frac{\partial^{2} u}{\partial x^{2}}+\frac{1-v}{2 a^{2}} \frac{\partial^{2} u}{\partial \theta^{2}}+\frac{1+v}{2 a} \frac{\partial^{2} v}{\partial x \partial \theta}+\frac{v}{a} \frac{\partial w}{\partial x}-\frac{\ddot{u}}{c_{L}^{2}}=0, \\
& \frac{1+v}{2 a} \frac{\partial^{2} u}{\partial x \partial \theta}+\frac{1-v}{2} \frac{\partial^{2} v}{\partial x^{2}}+\frac{1}{a^{2}} \frac{\partial^{2} v}{\partial \theta^{2}}+\frac{1}{a^{2}} \frac{\partial w}{\partial \theta}+\frac{h^{2}}{8 a^{4}} \frac{v}{1-v} \\
& \quad \times\left(\frac{\partial^{3} w}{\partial \theta^{3}}+\frac{\partial w}{\partial \theta}\right)-\frac{\ddot{v}}{c_{L}^{2}}=0, \\
& \frac{v}{a} \frac{\partial u}{\partial x}+\frac{1}{a^{2}} \frac{\partial v}{\partial \theta}+\frac{w}{a^{2}}+\beta^{2}\left(a^{2} \frac{\partial^{4} w}{\partial x^{4}}+2 \frac{\partial^{4} w}{\partial x^{2} \partial \theta^{2}}+\frac{1}{a^{2}} \frac{\partial^{4} w}{\partial \theta^{4}}\right. \\
& \left.\quad+\frac{4-v}{2(1-v)} \frac{1}{a^{2}} \frac{\partial^{2} w}{\partial \theta^{2}}+\frac{2+v}{2(1-v)} \frac{w}{a^{2}}\right)+\frac{\ddot{w}}{c_{L}^{2}}-\frac{p_{a}\left(1-v^{2}\right)}{E h} \\
& \quad=\frac{p_{0}\left(1-v^{2}\right)}{E h},
\end{aligned}
$$

where $\beta$ is the shell thickness parameter given by $\beta^{2}=h^{2} / 12 a^{2}, a$ is the mean radius of the shell, $h$ is the shell thickness, $v$ is the Poisson's ratio, $E$ is the Young's modulus, $c_{L}$ is the extensional phase speed of the shell material, $p_{a}(\theta, x)$ is the fluid loading acting normally to the cylindrical surface of the shell, the spectral equation of motion of the forced response of the system can be represented into a matrix form,

$$
\left[\begin{array}{lll}
L_{11} & L_{12} & L_{13} \\
L_{21} & L_{22} & L_{23} \\
L_{31} & L_{32} & L_{33}
\end{array}\right]\left[\begin{array}{c}
\bar{U}_{n} \\
\bar{V}_{n} \\
\bar{W}_{n}
\end{array}\right]=\left[\begin{array}{c}
0 \\
0 \\
a F_{0} e^{i k_{n} x_{0}} \\
\hline \sqrt{2 \pi} \rho_{s} c_{L}^{2} h / a
\end{array}\right],
$$

where the elements of the matrix system are

$$
\begin{aligned}
L_{11} & =-\Omega^{2}+\left(k_{n} a\right)^{2}+\frac{1}{2}(1-v) n^{2}, \quad L_{12}=\frac{1}{2}(1+v) n\left(k_{n} a\right), \\
L_{13}= & v\left(k_{n} a\right), \quad L_{21}=L_{12}, \\
L_{22} & =-\Omega^{2}+\frac{1}{2}(1-v)\left(k_{n} a\right)^{2}+n^{2}, \\
L_{23}= & n-\frac{h^{2}}{8 a^{2}} \frac{v}{1-v} n\left(n^{2}-1\right), \\
L_{31}= & L_{13}, \quad L_{32}=n, \\
L_{33}= & -\Omega^{2}+1+\beta^{2}\left(\left[\left(k_{n} a\right)^{2}+n^{2}\right]^{2}-\frac{4-v}{2(1-v)} n^{2}\right. \\
& \left.+\frac{2+v}{2(1-v)}\right)-\mathrm{FL} .
\end{aligned}
$$

In Eq. (9), $\boldsymbol{\Omega}$ is the nondimensional frequency $\Omega=\omega a / c_{L}$. FL is the fluid loading term due to the presence of the acoustic field and is given by ${ }^{1}$

$$
\mathrm{FL}=\Omega^{2}\left(\rho_{f} / \rho_{s}\right)(h / a)^{-1}\left(k^{r} a\right)^{-1}\left[J_{n}\left(k^{r} a\right) / J_{n}^{\prime}\left(k^{r} a\right)\right],
$$

where $\rho_{f}$ is the density of the fluid. The variable $k^{r} a$ is the nondimensional radial wave number that can be obtained from the wave-number vector relationship as

$$
k^{r} a= \pm\left[\Omega^{2}\left(c_{L} / c_{f}\right)^{2}-\left(k_{n} a\right)^{2}\right]^{1 / 2},
$$

where $c_{f}$ is the free wave speed of the fluid.

Solving for $\bar{W}_{n}$ and taking the inverse Fourier transform give the radial displacement

$$
\begin{aligned}
w(\Omega, x / a, n)= & \frac{F_{0} \cos (n \theta)}{2 \pi \rho_{s} c_{L}^{2} h / a} \int_{-\infty}^{+\infty} I_{33} \\
& \times \exp \left[i\left(k_{n} a\left|x / a+x_{0} / a\right|\right)\right] d k_{n} a,
\end{aligned}
$$

where

$$
I_{33}=\left(L_{11} L_{22}-L_{12} L_{21}\right) /(\operatorname{det}|L|)
$$

The solution of the integral in Eq. (12) has already been discussed by Fuller ${ }^{3}$ and is performed by the method of residues, each of these residues being evaluated at the poles, i.e., at the eigenvalues $k_{n s}$ of the infinite system. Note that, as the continuous integral of Eq. (12) is evaluated by a summation of residues over the branch number $s$, the subscript of the wave number $k_{n}$ has altered to $n s$.

\section{Optimal control}

Consider the second control approach on Fig. 2, with two control forces/two minimization points (the first case, one control force/one minimization point, being a simplified case of the other). Note that two error points are needed to avoid an overdetermined system. The total radial displacements of the shell at locations $X=A$ and $B$ can be expressed in matrix form by

$$
\left[w^{t}\right]=[D] W_{n s}^{\mathrm{inc}}+[C][F]
$$

where the matrices are given by 


$$
\begin{gathered}
{[D]^{T}=\left[\left.\left.\exp \left(i\left(k_{n s} a\right)_{\mathrm{inc}} \frac{\left(x+x_{i}\right)}{a}\right)\right|_{x=0} \exp \left(i\left(k_{n s} a\right)_{\mathrm{inc}} \frac{\left(x+x_{i}\right)}{a}\right)\right|_{x=\Delta X_{p}}\right],} \\
{[C]=\frac{1}{2 \pi \rho_{s} c_{L}^{2} h / a}\left[\begin{array}{l}
\left.\left.\int_{-\infty}^{+\infty} I_{33} \exp \left[i\left(k_{n} a\left|\frac{x+x_{0}}{a}\right|\right)\right] d k_{n} a\right|_{x=0} \quad \int_{-\infty}^{+\infty} I_{33} \exp \left[i\left(k_{n} a\left|\frac{x+x_{0}-\Delta X_{f}}{a}\right|\right)\right] d k_{n} a\right|_{x=0} \\
\left.\left.\left.\int_{-\infty}^{+\infty} I_{33} \exp \left[i\left(k_{n} a\left|\frac{x+x_{0}}{a}\right|\right)\right] d k_{n} a\right|_{x=\Delta x_{p}} \quad \int_{-\infty}^{+\infty} I_{33} \exp \left[i\left(k_{n} a\left|\frac{x+x_{0}-\Delta X_{f}}{a}\right|\right)\right] d k_{n} a\right|_{x=\Delta x_{p}}\right],
\end{array}\right.}
\end{gathered}
$$

$[F]^{T}=\left[\begin{array}{ll}F_{c 1} & F_{c 2}\end{array}\right]$,

$$
\left[w^{t}\right]^{T}=\left[\begin{array}{ll}
w^{t}(A) & w^{t}(B)
\end{array}\right],
$$

and []$^{T}$ denotes the matrix transpose operator.

In matrix form, the square of the displacement modulus is therefore

$$
\begin{aligned}
{\left[w^{t}\right]^{H}\left[w^{t}\right]=} & {\left[D W_{n s}^{\mathrm{inc}}+C F\right]^{\mathrm{H}}\left[D W_{n s}^{\mathrm{inc}}+C F\right] } \\
= & W_{n s}^{\text {inc }}\left[D^{\mathrm{H}} D\right] W_{n s}^{\mathrm{inc}}+F^{\mathrm{H}}\left[C^{\mathrm{H}} D\right] W_{n s}^{\mathrm{inc}} \\
& +W_{n s}^{\mathrm{inc}}\left[D^{\mathrm{H}} F\right] F+F^{\mathrm{H}}\left[C^{\mathrm{H}} C\right] F,
\end{aligned}
$$

where superscript " $H$ " denotes the Hermitian operator.

The optimal control forces to minimize this quadratic cost function are then given by ${ }^{9}$

$$
[F]_{\mathrm{opt}}=-\left[C^{\mathrm{H}} C\right]^{-1}\left[C^{\mathrm{H}} D\right] W_{n s}^{\mathrm{inc}} .
$$

\section{Transmission power coefficient}

Let us express the shell displacements and the pressure in the fluid field for a particular circumferential mode $n$ as a series of modes,

$$
\begin{aligned}
& u=\sum_{s=0}^{S_{n}} U_{n s} \cos (n \theta) e^{i\left(k_{n s} x-\omega t-\pi / 2\right)}, \\
& v=\sum_{s=0}^{S_{n}} V_{n s} \sin (n \theta) e^{i\left(k_{n s} x-\omega t\right)}, \\
& w=\sum_{s=0}^{S_{n}} W_{n s} \cos (n \theta) e^{i\left(k_{n s} x-\omega t\right)}, \\
& p=\sum_{s=0}^{S_{n}} P_{n s} \cos (n \theta) J_{n}\left(k_{s}^{r} r\right) e^{i\left(k_{n s} x-\omega t\right)} .
\end{aligned}
$$

Power flows in the $x$ direction in the fluid field, $P_{f}$, and in the shell wall, $P_{s}$, have already been derived in several references (Refs. 1, 2, and 4) and are given by

$$
\begin{aligned}
P_{f}= & \frac{\pi}{2} c_{L}^{3} \Omega^{3} \rho_{f} \epsilon \sum_{s=0}^{S_{n}} \sum_{j=0}^{S_{n}} W_{n s} \exp \left(i k_{n s} x\right) W_{n j}^{*} \\
& \times \exp \left(-i k_{n j}^{*} x\right) F_{s j n}^{f},
\end{aligned}
$$

where the fluid power factor is given by

$$
\begin{aligned}
F_{s j n}^{f}= & \frac{1}{a^{2}}\left(\frac{1}{k_{s}^{r} a J_{n}^{\prime}\left(k_{s}^{r} a\right)}\right) \\
& \times\left(\frac{k_{n j} a}{k_{j}^{r} a J_{n}^{\prime}\left(k_{j}^{r} a\right)}\right)^{*} \int_{0}^{a} J_{n}\left(k_{s}^{r} r\right) J_{n}^{*}\left(k_{j}^{r} r\right) r d r,
\end{aligned}
$$

and in which $\epsilon=2$ if $n=0$ and $\epsilon=1$ if $n \geqslant 1$;

$$
\begin{aligned}
P_{s}= & \pi \rho_{s} \mathcal{c}_{L}^{3} \Omega \epsilon \sum_{s=0}^{S_{n}} \sum_{j=0}^{S_{n}} W_{n s} \exp \left(i k_{n s} x\right) W_{n j}^{*} \\
& \times \exp \left(-i k_{n j}^{*} x\right) S_{s j n}^{f},
\end{aligned}
$$

where the shell factor $S_{s j n}^{f}$ is given by

$$
\begin{aligned}
S_{s j n}^{f}= & {\left[(h / a)^{3} / 12\right]\left[\left(k_{n s} a\right)^{2}\left(k_{n j}^{*} a\right)+v n^{2}\left(k_{n j}^{*}\right)+R_{a s}\left(k_{n s} a\right)\right.} \\
& \left.\times\left(k_{n j}^{*} a\right)+n R_{t s}\left(k_{n j}^{*} a\right)\right]+[(h / a) / 2] \\
& \times\left[\left(k_{n s} a\right) R_{a s} R_{a j}^{*}+n v R_{t s} R_{a j}^{*}+v R_{a j}^{*}\right]+[(h / a) / 4] \\
& \times(1-v)\left(n R_{a s} R_{t j}^{*}+k_{n s} a R_{t s} R_{t j}^{*}\right) .
\end{aligned}
$$

In Eq. (28), $\boldsymbol{R}_{a s}$ and $\boldsymbol{R}_{t s}$ are the ratios of axial and torsional to radial amplitudes of vibration obtained by resubstituting the derived axial wave number $k_{n s}$ into the equations of motion (9) of the free vibrating system. ${ }^{7}$ In Eqs. (25) and (27), the radial displacement amplitude $W_{n s}$ is obtained by substituting the optimal control forces into the Eq. (12).

The performance of the control discontinuity is then evaluated by the transmission power coefficient $T_{p}$ evaluated over the region of application of control as

$$
\begin{aligned}
T_{p} & =\frac{\text { transmitted power flow (in fluid \& shell) }}{\text { incident power flow (in fluid \& shell) }} \\
& =\frac{\left(P_{f}+P_{s}\right)_{\text {trans }}}{\left(P_{f}+P_{s}\right)_{\text {inc }}}
\end{aligned}
$$

The incident wave is assumed to have unit amplitude $W_{n s}^{\text {inc }}=1$. Note that this definition of control performance ignores the effect of the active control system on the source power itself. We assume that the source is unaffected by the application of active control.

Power transmisson loss is then defined as

$$
\mathrm{TL}=10 \log _{10}\left(T_{p}\right) .
$$


TABLE I. Material properties.

\begin{tabular}{lccccc}
\hline \hline & $\begin{array}{c}\text { Young's } \\
\text { modulus } \\
\left(\mathrm{N} / \mathrm{m}^{2}\right)\end{array}$ & $\begin{array}{c}\text { Poisson's } \\
\text { ratio }\end{array}$ & $\begin{array}{c}\text { Density } \\
\left(\mathrm{kg} / \mathrm{m}^{3}\right)\end{array}$ & $\begin{array}{c}\text { Free wave } \\
\text { speed } \\
(\mathrm{m} / \mathrm{s})\end{array}$ & $\begin{array}{c}\text { Damping } \\
\text { ratio }\end{array}$ \\
\hline Steel & $19.2 \times 10^{10}$ & 0.3 & 7800 & 5200 & 0.02 \\
Water & $\cdots$ & $\cdots$ & 1000 & 1500 & $\cdots$ \\
\hline \hline
\end{tabular}

\section{RESULTS}

\section{A. Breathing mode $n=0$}

\section{Shell thickness $h / a=0.05$}

Results are given for two cases of discontinuity, when one control line force is applied and the radial displacement of the shell is minimized at one location, and when two control forces spaced a distance $\Delta X_{f}=0.1 a$ are used to minimized the radial displacement at two different axial locations spaced a distance $\Delta X_{p}=0.05 a$. In the second case the two forces have been spaced a distance about 10 times smaller than the minimum wavelength, at the highest frequency. In this situation, the forces introduce a line moment component in the pipe shell in addition to a radial force. To evaluate the radial displacement, using the residues theorem, eight roots had to be considered to reach convergence of the expression in Eq. (12). Material properties for the system are given in Table I. Note that an artificial damping $\eta$ has been added to the shell material, making $E$, the Young modulus, complex and equal to $E^{\prime}=E(1-\eta i)$.

Figure 5 shows the power transmission loss TL considering an in vacuo steel shell of thickness $h / a=0.05$. The branch $s=1$ of the dispersion curves in Fig. 3 was used as an incident disturbance wave corresponding to a structural source. At low frequencies $(\Omega \leqslant 0.95)$, even though the incident wave is extensional in nature, it is possible to reduce the downstream power flow because of the Poisson's ratio effect. Attenuations vary from 10 to $27 \mathrm{~dB}$ with the one control force/one error point configuration and attenuations from 10 to $40 \mathrm{~dB}$ are obtained the two control forces/

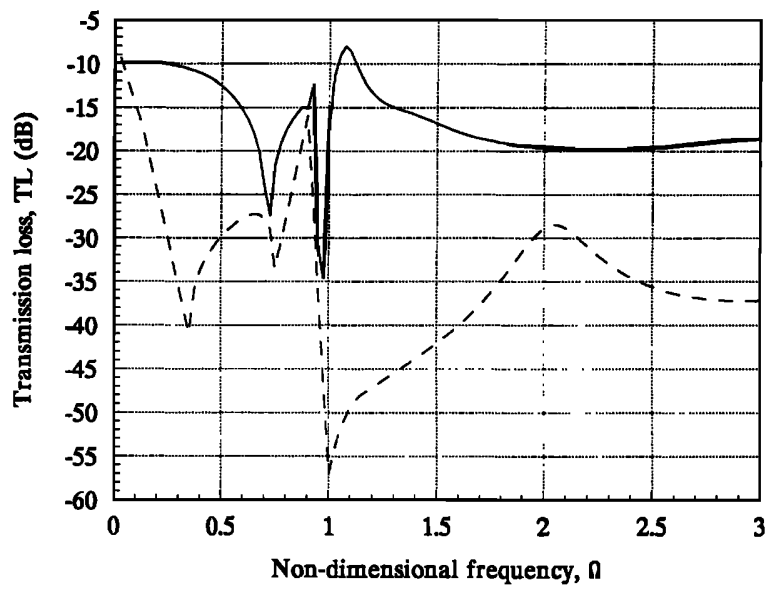

FIG. 5. Transmission power coefficient, in vacuo steel shell $h / a=0.05$, $n=0$, branch $s=1$ incident;- - , one control force;-----, two control forces.

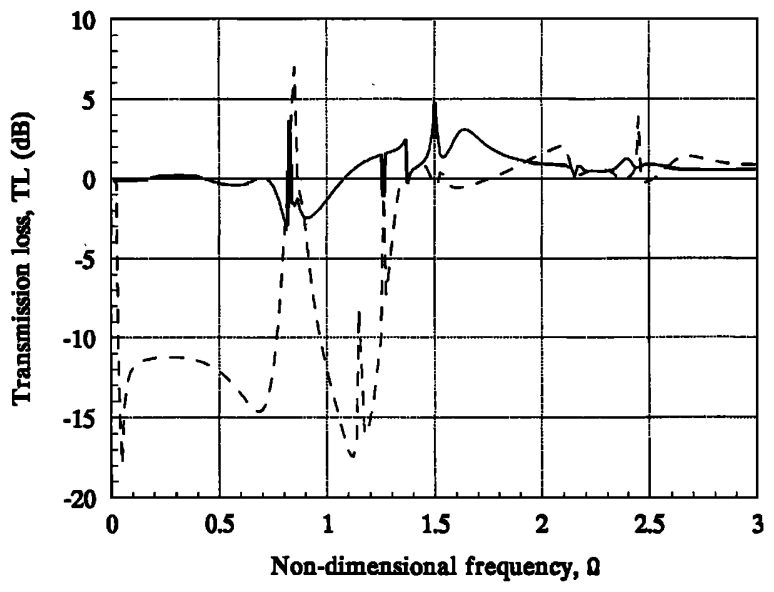

FIG. 6. Transmission power coefficient, water-filled steel shell, $h / a$ $=0.05, n=0$, branch $s=2$ incident; - $-\longrightarrow$, one control force;----, two control forces.

two error points configuration. Above the ring frequency $\Omega=1$, the incident wave changes in nature to a flexural wave and is thus more efficiently affected by radial line forces. About $20 \mathrm{~dB}$ of attenuation are obtained with one force at frequencies above $\Omega=1.5$. With two closely spaced control forces, the total power flow is reduced from 30 to $50 \mathrm{~dB}$; the out-of-plane and part of the in-plane vibrations have been strongly reduced.

When the shell is filled with water, two types of disturbance, corresponding to the two real branches on the dispersion curves [Fig. 4(a)], can be considered: a structural and a fluid type wave. Figure 6 shows the power transmission loss when the incident wave is the branch denoted as $s=2$, which is a structural type wave at low frequencies. Even though the radial displacement is perfectly controlled at the error point (for example, see the cases $\Omega=0.5$ and $\Omega=3$ in Fig. 7), one control force proves to be a very inefficient discontinuity, as no significant reduction of the transmitted power flow is obtained at any

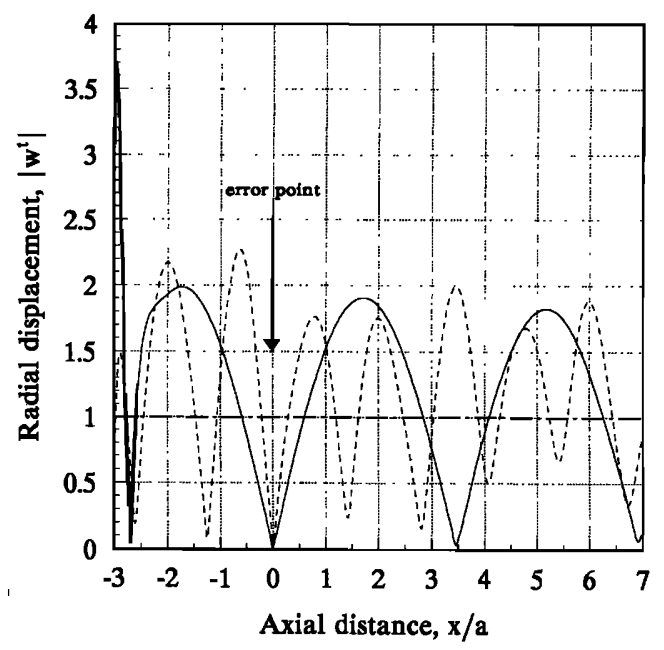

FIG. 7. Magnitude of the radial displacement of the wall, one control force, water-filled steel shell, $h / a=0.05$, branch $s=2$ incident;- - $\Omega=0.05 ;---, \Omega=3$; - - - , uncontrolled disturbance magnitude. 


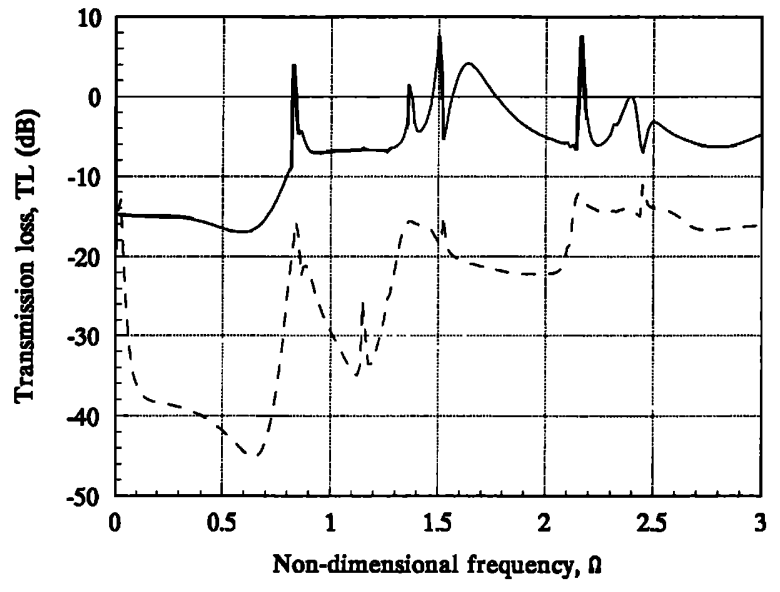

FIG. 8. Transmission power coefficient, water-filled steel shell, $h / a$ $=0.05, n=0$, branch $s=1$ incident;- $-\longrightarrow$, one control force;-----, two control forces.

frequency. The reason for this behavior is that the fluid modifies the energy propagation path at the control point and decreases the control performance by flanking energy around the discontinuity. Using two radial control forces, attenuations varying from 10 to $17 \mathrm{~dB}$ appear in the frequency range $\Omega \in[0.1 ; 1.3]$, except around the frequency $\mathbf{\Omega}=\mathbf{0 . 8 5}$ at which the coupled system strongly resonates. For frequencies below $\Omega=0.05$, the distance between the control forces or the error points approaches zero with respect to the wavelength of the incident wave $(\lambda \simeq 30 a)$; therefore, results with one or two control forces become similar. No attenuation is achieved at frequencies above $\Omega \simeq 1.3$ as the incident wave turns into a pure fluid-type wave, very close to a pressure release duct solution, and most of the energy propagates via the fluid path. Note that, for some frequencies associated with resonances of the system, the transmission loss is positive, implying that although the radial vibration is minimized at the error points the total energy flow has increased.

Figure 8 displays the power transmission loss when the incident disturbance is a fluid-type wave, i.e., the branch denoted as $s=1$ on the dispersion curves of Fig. 4(a). As mentioned before, the most important characteristic of this wave is to be pressure near field at the shell wall at all frequencies. Performing the control with only one force, we can see the surprising result that the propagated power flow is reduced over almost the whole frequency range; good attenuations of $15 \mathrm{~dB}$ are obtained at low frequencies and an average $6 \mathrm{~dB}$ occurs above the frequency $\Omega=0.7$. Because of breathing resonances of the coupled system in the radial direction, an increased power flow appears at a few discrete frequencies such as $\boldsymbol{\Omega}=0.85$. Using two control forces, more noticeable attenuations of the total power flow are obtained, varying from 14 to $45 \mathrm{~dB}$. The explanation for this unexpected result is in the particular nature of the incident wave; the near field closely hugging the wall reacts like a mass loading and is thus strongly affected by structural forces.

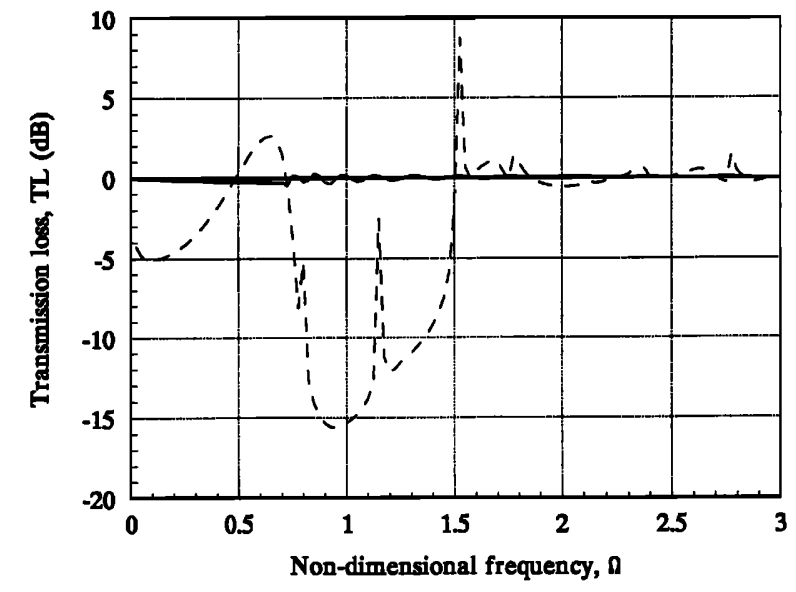

FIG. 9. Transmission power coefficient, water-filled steel shell, $h / a$ $=0.005, n=0$, branch $s=2$ incident;-_ —, one control force;-----, two control forces.

\section{Shell thickness $h / a=0.005$}

Figures 9 and 10 show the power transmission loss when the discontinuities consisting of one or two control forces (same configuration as in Sec. II A 1) are applied to a fluid-filled steel shell of thickness $h / a=0.005$. Considering the structural-type wave $s=2$ as a disturbance, in Fig. 9 , we can see that power flow reductions can not be achieved using one control force only. Using two control forces, good performances appear in the frequency range $\Omega \in[0.7 ; 1.5]$. In this range, up to $16 \mathrm{~dB}$ of attenuation is obtained at the frequency $\Omega=0.95$. Poor performances occur above this range because of the coupling between the two media (see Sec. II A 1). It was expected that below $\Omega=0.7$, the line moment created by the two control forces would be more effective on the in-plane propagation as the shell compliance is increased. In reality, performances in the low-frequency range have been severely decreased. A maximum of 5-dB reduction is obtained at the frequency $\boldsymbol{\Omega}=\mathbf{0 . 1}$. This result can be explained by the fact that, as the shell compliance is increased, so is the coupling between

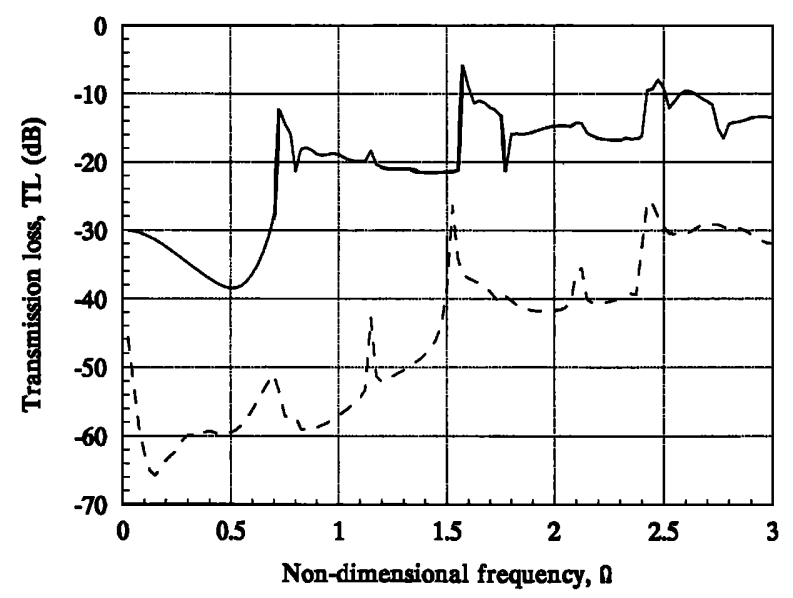

FIG. 10. Transmission power coefficient, water-filled steel shell, $h / a$ $=0.005, n=0$, branch $s=1$ incident;- $-\longrightarrow$, one control force;-----, two control forces. 


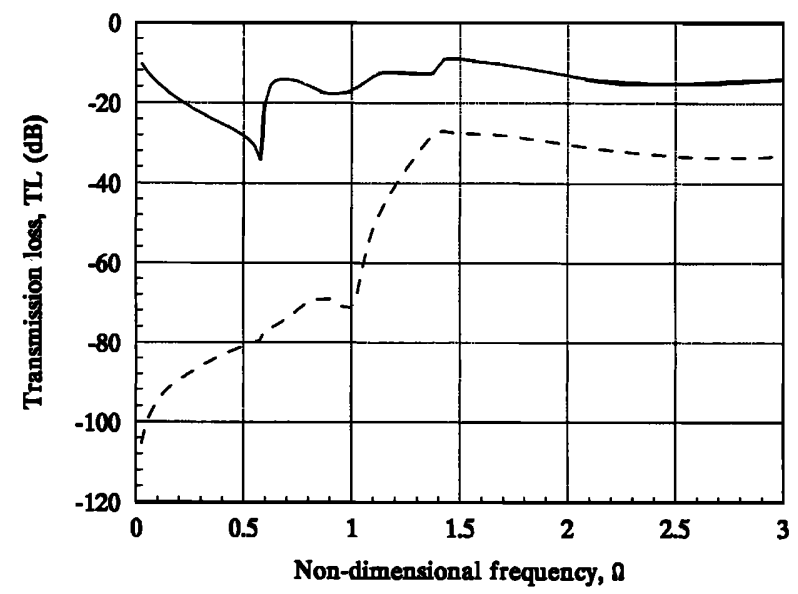

FIG. 11. Transmission power coefficient, in vacuo steel shell, $h / a=0.05$, $n=1$, branch $s=1$ incident;- - - , one control force;-----, two control forces.

the two media. That implies that the energy propagation path can be further modified, i.e., more energy can be flanked into the fluid medium.

When the shell thickness is decreased to a ratio $h / a$ $=0.005$, the fluid-type wave, i.e., the branch $s=1$, becomes increasingly subsonic and almost all the incident energy is concentrated near the shell wall. Therefore, this wave is more strongly affected by structural forces. With one control force, attenuations of the total power flow varying from 10 to $38 \mathrm{~dB}$ are obtained (see Fig. 10). Using the two control forces/two error points configuration, these attenuations reach levels varying from 30 to $66 \mathrm{~dB}$, which is approximately $20 \mathrm{~dB}$ more than with $h / a=0.05$.

\section{B. Beam mode $n=1$}

Figure 11 shows the power transmission loss for the two control configurations described in Fig. 2 considering an in vacuo shell of thickness $h / a=0.05$ vibrating in the $n=1$ circumferential mode with the $s=1$ wave incident. At low frequencies, the nature of the incident wave ${ }^{7}$ causes the pipe to vibrate as a long, slender beam. Therefore, performing the control with only one structural force provides good attenuations from 10 to $30 \mathrm{~dB}$ of the total power flow. At higher frequencies, the incident wave becomes almost purely flexural ${ }^{7}$ and is thus also highly affected by a structural force; reductions varying from 4 to $28 \mathrm{~dB}$ (at $\Omega=3$ ) are obtained. Of course, a pair of control forces is even more effective as it also reduces part of the in-plane propagation. At low frequencies, virtually total control of the disturbance is achieved (up to $100 \mathrm{~dB}$ of attenuation). At higher frequencies, an average $20 \mathrm{~dB}$ of attenuation has added to the results of the previous one force/one error point configuration.

When the shell is filled with water (Fig. 12), two important phenomena affect the control performance. At low frequencies, below the first acoustic cutoff frequency near $\Omega=0.55$, the control effectiveness is increased; up to $60 \mathrm{~dB}$ of attenuation is obtained with only one control force. In this frequency range, the fluid simply acts as an additional

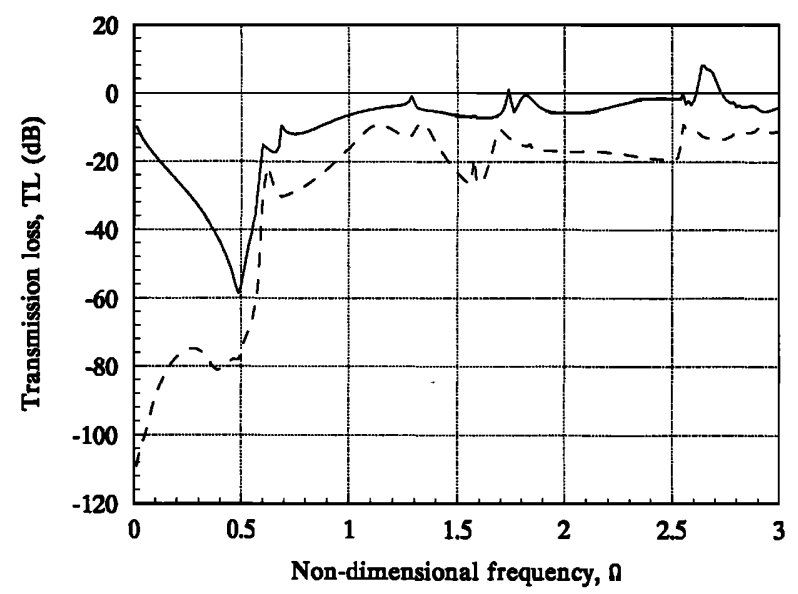

FIG. 12. Transmission power coefficient, water-filled steel shell, $h / a$ $=0.05, n=1$, branch $s=1$ incident; $--\longrightarrow$, one control force;-----, two control forces.

mass decreasing the fluid-filled pipe compliance. ${ }^{10}$ However, above the first cutoff frequency, as higher order àcoustic modes propagate, control performances are severely decreased; less than $5 \mathrm{~dB}$ of attenuation is obtained with one force and a maximum of $20 \mathrm{~dB}$ is achieved with two forces. More of the propagating energy is carried by the fluid medium and is thus weakly affected by structural forces.

\section{CONCLUSIONS}

The active control of wave propagation in fluid-filled elastic cylindrical shells has been analytically investigated. Control was applied as radial line forces while radial shell vibration was minimized at up to two downstream points.

In the $n=0$ case, although the control was structural, it has been noticed that it is surprisingly easier to reduce the total power flow propagating along the coupled system when the disturbance is a fluid-type incident wave. This behavior is associated with the particular nature of the wave, i.e., pressure near field close to the shell wall. For structural-type wave propagation, because of the coupling between the two media, the fluid severely decreases the control performance. Nevertheless, with this kind of disturbance, relatively good attenuations of the total power flow can be obtained in the non-dimensional frequency range $\Omega \in[0.7 ; 1.5]$ as the shell thickness is decreased.

In the $n=1$ case, it has been noticed that the fluid increases the control performances below the first acoustic cutoff frequency. Above this frequency, good control of the propagating power flow using structural forces is difficult to achieve as more energy is carried by the fluid medium due to acoustic waves cutting on and propagating, leading to flanking around the discontinuity provided by the control system.

\section{ACKNOWLEDGMENTS}

The authors gratefully ackowledge the Office of Naval Research for supporting this work. 
${ }^{1}$ C. R. Fuller and F. J. Fahy, "Characteristics of wave propagation and energy distribution in cylindrical elastic shells filled with fluid," J. Sound Vib. 81, 501-518 (1982).

${ }^{2}$ B. J. Brévart and C. R. Fuller, "Effect of an internal flow on the distribution of vibrational energy in an infinite fluid-filled thin cylindrical elastic shell," to appear in J. Sound Vib. 163(3) (1993).

${ }^{3}$ C. R. Fuller, "The input mobility of an infinite circular cylindrical elastic shell filled with fluid," J. Sound Vib. 87, 409-427 (1983).

${ }^{4} \mathrm{C}$. R. Fuller, "Monopole excitation of vibrations in an infinite cylindrical elastic shell filled with fluid," J. Sound Vib. 96(1), 101-110 (1984).

${ }^{5}$ G. F. Leyrat and J. M. Cushieri, "Effects of an internal flow on the vibration of infinitely long cylindrical shells," submitted to J. Acoust. Soc. Am (1992).
${ }^{6}$ B. J. Brévart and C. R. Fuller, "Radial impulsive excitation of infinite fluid-filled elastic cylindrical shells," accepted for publication in J. Sound Vib. (1993).

${ }^{7} \mathrm{C}$. R. Fuller, "The effects of wall discontinuities on the propagation of flexural waves in cylindrical shells," J. Sound Vib. 75, 207-228 (1981).

${ }^{8}$ E. H. Kennard, "The new approach to shell theory: Circular cylinders," J. Appl. Mech. 20, 33-40 (1953).

${ }^{9}$ P. A. Nelson, A. R. D. Curtis, S. J. Elliot, and A. J. Bullmore, "The minimum power output of free field point sources and the active control of sound," J. Sound Vib. 116(3), 397-414 (1987).

${ }^{10} \mathrm{P}$. H. White and R. J. Sawley, "Energy transmission in piping systems and its relation to noise control," Trans ASME, J. Eng. Ind. 94, 746751 (1972). 